\title{
ZOFÍO. HACIENDO FRENTE A LA \\ COVID-19 EN RED EN UN BARRIO
}

\author{
JOSÉ ÁNGEL ORTUÑO TORRES ${ }^{1}$ \\ JOSÉ ANTONIO JIMÉNEZ JIMÉNEZ²
}

\begin{abstract}
RESUMEN: Previamente a la pandemia de covid-19, la situación social de la comunidad de Madrid no estaba en correlación directa a su situación económica. entre otros hechos, hay que destacar que la desigualdad era la más alta de España (FOESSA, 2019), a esto, había que sumar la precaria situación de los servicios sociales en la comunidad de Madrid (AEDGSS, 2019). la pandemia no ha hecho sino agravar la situación social de numerosos vecinos y vecinas de Madrid, y sobrecargar la red pública de servicios sociales. Pero puso también de manifiesto, la capacidad de solidaridad y respuesta a los problemas sociales causados por la COVID-19 de las asociaciones de vecinos de diferentes barrios de la ciudad, siguiendo su larga trayectoria en defensa de la democracia, la vivienda o los equipamientos. Un ejemplo de la colaboración entre administraciones públicas, tercer sector y movimiento vecinal lo encontramos en el barrio de Zofío, del distrito de Usera (Madrid), en el reparto de alimentos durante el confinamiento. Esta experiencia nos lleva a preguntarnos si, en la solución de los problemas sociales que afronta la sociedad madrileña, se han explorado suficientemente las vías que ofrece el trabajo comunitario entre administraciones públicas, tercer sector y movimiento vecinal.
\end{abstract}

PALABRAS CLAVE: trabajo comunitario; movimiento vecinal.

\section{Zofío. Coping with COVID-19 in a neighborhood community}

ABSTRACT: Prior to the COVID-19 pandemic, an imbalance between the social reality and the economic situation of the autonomous region of Madrid could be perceived. for instance, the inequality index in Madrid was the highest in Spain (FOESSA, 2019), combined with a precarious situation of the social services in the region of Madrid (AEDGSS, 2019). The pandemic has aggravated the social situation of many residents of Madrid and overloaded the public network of social services. However, the COVID-19 pandemic has also highlighted the solidarity and problem-solving capacity of the different neighborhood associations of the city, in line with their long-standing defense of democracy, housing or facilities. An example of collaboration between public administrations, the third

1 Cáritas Diocesana de Madrid. Correo electrónico: jose.ortuno@caritasmadrid.org.

2 Cáritas Diocesana de Madrid. Correo electrónico: jose.jimenez@caritasmadrid.org. 
sector and a neighborhood movement can be found in the Zofío community, in the district of Usera (Madrid), with regard to the distribution of food during the confinement. This experience raises the question whether the opportunities of collaborations between public administrations, the third sector and the neighborhood movement have been sufficiently explored as possible solutions of the social problems faced by Madrid society.

KEY WORDS: community work; neighborhood movement.

Este breve trabajo no es la presentación preliminar de los resultados finales de unos resultados de investigación. Por el contrario, es un punto de partida de un trabajo de investigación sobre el trabajo en red y el trabajo comunitario como mecanismos de respuesta a problemas sociales que, en nuestra opinión, no encontrarán respuestas satisfactorias en el modelo establecido de provisión de servicios sociales en nuestra ciudad.

De manera introductoria, hay que recordar que la situación social de la ciudad de Madrid viene marcada por un profundo desequilibrio territorial, entre los distritos y barrios que la componen. La dinámica del desarrollo urbano, lejos de ser homogéneo, ha dado lugar a barrios en situación de vulnerabilidad. Vulnerabilidad que ha de entenderse como el potencial riesgo de que la población de estas zonas sea afectada por determinados problemas socioeconómicos. Existe la seria posibilidad de que el riesgo se convierta en una realidad estructural, sino lo ha hecho ya en algunos casos.

El Ayuntamiento de Madrid y la Universidad Carlos III de Madrid, utilizando la metodología Analytic Hierarchy Process, han desarrollado un Índice de Vulnerabilidad Territorial de barrios y distritos de Madrid, así como un ranking basado en dicho índice Ayuntamiento de Madrid (Ayuntamiento de Madrid, 2018). En la construcción de índice se tuvieron en cuenta variables relativas a la población (número de habitantes, tasa de inmigración), estatus socioeconómico (renta media del hogar), actividad económica (tasa de paro, parados mayores de 45 años, parados sin prestación), desarrollo urbano (valor catastral de las viviendas) y necesidades asistenciales (tasa de demanda de dependientes, familias perceptoras de renta mínima, tasa de servicios asistenciales a domicilio, tasa de teleasistencia). Los resultados muestran la presencia de un fuerte desequilibrio en la ciudad de Madrid, existiendo una línea recta que va del distrito de Carabanchel al de San Blas, por debajo de la cual se encuentran los distritos en peor situación. Puente de Vallecas, Usera y Villaverde son los tres distritos que peores resultados arrojan. La existencia de esta línea y de los desequilibrios que representa, supone una violación del lefebvriano Derecho a la Ciudad (Uceda, 2016). No es una cuestión baladí, teórica: afecta directa y negativamente a la calidad de vida de las personas 
que viven en esas zonas vulnerables, cuando no directamente a su esperanza de vida (Benach y Muntaner, 2005).

Establecida la existencia de un desequilibrio territorial dentro de la ciudad de Madrid, recogemos brevemente cuál la situación de exclusión y desarrollo social en la Comunidad de Madrid, previa a la COVID-19. Según el VIII informe FOESSA (FOESSA, 2019), en los cinco años previos a la publicación, habían mejorado los niveles de integración social de la población. La población en situación de inclusión social había pasado del 37\% al 50\%, mientras que la exclusión social se había reducido en un $25 \%$ entre 2013 y 2018. A pesar de esta mejoría, dentro de la franja de inclusión, había 900.000 personas en situación vulnerable, y un millón de personas en situación de exclusión social: el 16,2\% de la población. De ellas, 490.000 sufrían exclusión severa y 269.000 exclusión social extrema. Ser mujer, estar desempleado, se inmigrante extracomunitario, carecer de estudios o vivir en un municipio de menos de 5.000 habitantes, son factores que incrementan el riesgo de exclusión social. El riesgo es mayor, asimismo, en los hogares monoparentales y en los que están formados por cinco o más personas. Otros aspectos que ensombrecen el incremento del número de personas en situación de inclusión social es que, en la Comunidad de Madrid, la desigualdad entre el 20\% más rico y el 20\% más pobre es la más alta de España. El empleo, la vivienda y la salud son los principales factores creadores de exclusión.

Previamente a la pandemia, ¿cuál era la situación de los servicios sociales? En 2018 los Servicios Sociales de la Comunidad de Madrid, según el índice DEC de la Asociación Estatal de Directores y Gerentes en Servicios Sociales, ocupaban el último puesto en la clasificación del grado de desarrollo de los servicios sociales por Comunidades Autónomas. De hecho, se constataba un estancamiento desde 2015, que, en algunos aspectos, eran un retroceso.

Esta situación tenía varias explicaciones. En primer lugar, nuestra Comunidad carece de un marco legal que reconozca derechos subjetivos en materia de servicios sociales. Esto implica que no haya fundamento jurídico alguno para la oferta de servicios y prestaciones del Sistema de Servicios Sociales en Madrid. En segundo lugar, el gasto por habitante es un 10\% inferior a la media estatal. Por último, la red de atención primaria de ayuntamientos y comarcas es exigua, con un solo profesional por cada 8.354 habitantes, cuando la media estatal es de uno por cada 2.348 (AEDGSS, 2019).

Con este punto de partida, no es extrañar que, con la pandemia, los servicios sociales municipales se hayan visto desbordados, al punto de que la prensa se hizo eco de la situación (Torres, 2020) y surgieron las respuestas vecinales que motivan este trabajo. El Instituto Nacional de Administración Pública (INAP), ha financiado un estudio (Calzada, 2020) de varias 
universidades sobre la respuesta de los servicios sociales públicos ante la pandemia. Los resultados son descriptivos, y no están desglosados por comunidades autónomas, por lo que no podemos conocer de manera concreta, la situación y la capacidad de respuesta de los servicios sociales madrileños. Volviendo a las fuentes periodísticas (Negro, 2021), en mayo de 2021, nueve de los 21 distritos de la ciudad tenían menos trabajadores sociales que los que el propio Ayuntamiento recoge en su carta de servicios (un trabajador social por cada 6.000 habitantes). La ratio del Ayuntamiento está lejos de la recomendada por el Colegio Oficial de Trabajo Social (un trabajador social por cada 3.000 habitantes) y del que sería el número óptimo, un trabajador social por cada 1700 habitantes (AEDGSS, 2019). En Madrid, habría que triplicar los 529 trabajadores sociales actuales. El personal de los servicios sociales municipales ya era insuficiente antes de la pandemia.

El refuerzo que han supuesto la contratación, por parte del Ayuntamiento de Madrid, de 114 trabajadores sociales y 41 auxiliares administrativos, es insuficiente. En primer lugar, porque la introducción de medidas altamente necesarias, como la tarjeta de familias, incrementa notablemente la carga burocrática que han afrontar estos profesionales. Por otro lado, el mencionado informe del INAP demuestra el incremento notable de demanda sufrido por estos servicios, con un volumen sensible de personas que nunca antes habían sido atendidas por los servicios sociales, sin tener que dejar de atender a las personas que ya atendían con anterioridad. El propio Ayuntamiento ha emitido un informe sobre las consecuencias sociales de la crisis de la COVID-19, que no augura un descenso del número de personas que son o serán atendidas por los servicios sociales ni del número de atenciones (Ayuntamiento de Madrid, 2020). De la misma manera, Cáritas Española ha publicado un estudio (Cáritas Española, 2021), sobre una muestra de 4.000 hogares que ya recibían acompañamiento por su parte. Se constata un agravamiento de su situación: no hay una verdadera recuperación laboral; crece el número de hogares sin ingresos; el 16\% de los encuestados ha tenido que cambiar de domicilio; se constata un serio problema de brecha digital; ha empeorado el estado de salud de este colectivo, especialmente su salud mental.

Ante esta situación, con un incremento de los problemas sociales y unos mecanismos públicos de respuesta desbordados, ¿qué se puede hacer?

En nuestra opinión, hay que potenciar la respuesta comunitaria, iniciando o consolidando la construcción de redes público-privadas que permitan llevar a cabo acciones de desarrollo y de trabajo comunitario que, de otra manera, no se podría llevar a cabo. La acción del movimiento vecinal madrileño durante la pandemia, nos lleva a pensar que la red de asociaciones de vecinos podría ser un buen puntal. 
En la actualidad, en el ámbito de la Comunidad Autónoma de Madrid y agrupadas en la Federación Regional de Asociaciones de Vecinos de Madrid, hay casi 300 asociaciones vecinales. La acción que llevan a cabo, como en el pasado es muy amplia: empleo, medioambiente, seguridad ciudadana, urbanismo y vivienda, transporte, consumo, sanidad o participando en iniciativas institucionales como los Planes Integrales de Barrio.

Las asociaciones de vecinos tienen un largo recorrido. Conocido es que el régimen franquista restringió severamente los derechos de reunión y asociación. No obstante, a comienzos de los años sesenta, el régimen flexibilizó esta política de cierre. El primer paso fue la Orden de 24 de junio de 1963, que regulaba la constitución de las asociaciones de cabezas de familia. Este hecho, aceleró un debate que dio lugar a una verdadera ley de asociación, promulgada el 24 de diciembre de 1964, seguidas de su Reglamento y unas Disposiciones complementarias del 10 de julio de 1965. Aprobado este parco marco de participación, las asociaciones de cabezas de familia sirvieron de germen de las asociaciones de vecinos (Caprarella, 2016). En torno a ellas, los vecinos y las vecinas iniciaron y protagonizaron la lucha contra el urbanismo y la política local del desarrollismo franquista. Será durante el período que va de 1975 a 1978 cuando cristalicen las luchas vecinales, inicialmente contra las contradicciones creadas por una etapa caracterizada por el crecimiento a toda costa. Si bien los bajos costes de las viviendas, garantizados por los promotores públicos y privados, mediante una mezcla de corrupción e incompetencia se convierten en altos costes sociales para las vecinas y los vecinos. Viviendas sí, pero ubicadas en zonas sin urbanizar y sin ni siquiera los equipamientos más básicos.

Un relato de esta etapa lo hace Manuel Castells (Castells, 1986). El movimiento vecinal tuvo una fuerte presencia y actividad en la ciudad de Madrid, constituyéndose en escuela de ciudadanía y participación:

Aunque el movimiento ciudadano estuvo presente en todo el Estado español, en Madrid cobró una significación especial por su proximidad a la cúspide del poder, en donde se libraban las batallas para decidir hasta dónde podía llegar la naciente democracia (...) Por eso el movimiento ciudadano madrileño tuvo un impacto decisivo al extender el clamor por la democracia a sectores sociales sin experiencia de lucha política y al convertir el espacio de la vida cotidiana de la capital en un espacio de debate, en donde el ordeno y mando ya no funcionaba. (Castells, 1986).

A esta función, bien acreditada (Díaz, 1999; Gómez, 2017; Hedo, 2010), como escuela de ciudadanía y participación democrática (Díaz, 1999; Gómez, 2017), se suman otras muchas labores desarrolladas por el movimiento vecinal desde sus orígenes. Las reivindicaciones iniciales del movimiento vecinal 
tuvieron que ver con el urbanismo y la dotación de infraestructuras y dotaciones de todo tipo en los barrios periféricos de la ciudad: desde aceras y asfaltado de calles, a la construcción de centros educativos o zonas verdes. También la lucha contra la carestía de la vida, en concreto contra el fraude en el peso del pan, fue otro de los ámbitos reivindicativos del movimiento vecinal (Caprarella, 2016). El carácter elemental de los elementos objetivo de reclamación lo resume muy bien el lema de la asociación vecinal de Orcasitas: del barro al barrio. El urbanismo, en especial en lo tocante a la participación vecinal en su planificación (Bonet, 2012) y la creación de espacios de relación informal que dan respuestas a necesidades de las vecinas y los vecinos y que o bien escapan a los mecanismos del mercado o bien ése y las administraciones públicas son incapaces de satisfacer (Jiménez, 2013) han sido otras laborales del movimiento vecinal. Sobre esta segunda, la de crear mecanismo de cobertura ajenos al mercado y a las administraciones públicas, volveremos enseguida.

Si el movimiento vecinal fue el primer espacio de participación social de muchos hombres, lo cierto es que jugó un papel importantísimo como mecanismo de incorporación de las mujeres al ámbito asociativo (Bustos, 2006; Morell, 2012). Mujeres fueron las protagonistas de las movilizaciones contra la carestía de la vida o en reclamación de equipamientos educativos (Caprarella, 2016). Alcanzadas las reivindicaciones básicas, la acción de las asociaciones de vecinos y su fuerza fue menguando a lo largo de la década de los 80 del siglo XX, así como mutando sus objetivos: la inseguridad ciudadana (Cañedo, 2011); el desempleo (Caprarella, 2016); la integración de los nuevos vecinos y vecinas inmigrantes, el medioambiente (Escudero, 2020; García, 2021); la turistificación (Ruano, Iglesias y Polo, 2019); la gentrificación y la especulación (Limón, 2015; Morell, 2013); o la defensa de los servicios públicos (Manahan, 2020).

Hemos visto los desequilibrios estructurales de la ciudad de Madrid y la situación social de la Comunidad de Madrid, previa a la crisis de la COVID-19; cómo ha afectado la pandemia a las familias previamente atendidas por Cáritas Madrid. También hemos visto la situación de los Servicios Sociales y su capacidad de respuesta en esta situación. Todos factores, que confluyen con una larga tradición asociativa vecinal, con esta amplia implantación territorial y la larga historia de movilización y la capacidad de gestión de recursos públicos (mediante acuerdos, convenios, contratos, etc.) y de autogestión, no es de extrañar que llegada la pandemia de la COVID-19, y pasado los primeros momentos de estupor e incertidumbre, las asociaciones vecinales respondieran a las necesidades de las vecinos y lo vecinos de Madrid (FRAVM, 2020; Rochet, 2020). Hay casos que se han hecho mediáticamente famosos, como el de Somos Tribus, en Vallecas, que ha recibido el Premio Ciudadano Europeo 
2020 concedido por el Parlamento Europeo. También la asociación de vecinos de Latina, con un intenso reparto de alimentos, ha logrado eco mediático. Esta asociación reparte unas 30 toneladas de alimentos mensuales y se ha negado a colaborar con los Servicios Sociales del Ayuntamiento de Madrid.

También en el distrito de Usera, cuyos indicadores socioeconómicos en 2020, se resumen diciendo que es el tercer distrito de los 21 existentes en el ranking de vulnerabilidad y sus siete barrios entre los veinticinco más vulnerables de los 132 barrios de la ciudad, varias entidades vecinales han puesto en marcha iniciativas de apoyo mutuo (Artigas, 2020), al margen de la colaboración con las administraciones públicas. En Zofio, uno de los siete barrios que constituyen el distrito, la asociación vecinal puso en marcha un modelo que, en nuestra opinión, es un ejemplo de cómo, en el futuro han de afrontarse los problemas sociales.

Desde hace diez años, la Asociación Vecinal Zofío trabaja en red con todas las entidades sociales, religiosas y los recursos públicos presentes en el barrio. La Asociación dinamiza la Mesa de Zofío, coordinadora en el que están presentes veinte entidades sociales y recursos públicos. A raíz de la COVID-19, este foro puso en marcha tres grupos de trabajo: uno de salud mental, para evaluar el impacto que la pandemia y el confinamiento ha tenido sobre la población infantil del barrio; un grupo sobre brecha digital, porque la situación que vivimos ha puesto de manifiesto la existencia de un importante problema de brecha digital, nueva dimensión de la pobreza, y que dificulta o imposibilita el acceso a la administración digital; y un grupo de ocio, con la misma motivación que el grupo de brecha digital.

Pero eso ha sido ya en 2021. En 2020, la acción de la Asociación Vecinal Zofío, en lo tocante a las consecuencias de la pandemia, se centró en el reparto de alimentos y en el apoyo a aquellos vecinos y vecinas (más de 150) con dificultades para gestionar el Ingreso Mínimo Social.

En cuanto al reparto de alimentos, el modelo de intervención se basó en el trabajo en red. Se establecieron un punto de reparto de alimentos. Uno en la propia asociación de vecinos de Zofío y otro en la parroquia de San Juan de Ávila. Las personas que recibían alimentos eran derivadas por las profesoras técnicas de servicios a la comunidad de los centros educativos y las trabajadoras de los servicios sociales municipales, así como por el personal de otras entidades del tercer sector presentes en el barrio. Se estableció, además, coordinación con las personas encargadas del reparto de alimentos en la parroquia San Juan de Ávila, para evitar duplicidades. También se atendió a personas que acudían a la asociación directamente, pero además de ser atendidos eran derivados a los Servicios Sociales municipales, y se establecía la correspondiente coordinación con el resto de la red. El trabajo en red, permitía, por 
tanto, que entraran en juego profesionales de la intervención social que podría hacer diagnósticos precisos y diseñar intervenciones integrales, por un lado; mientras que la parte asociativa proporcionaba alimentos de manera rápida y facilitaba el trámite de la gestión de Ingreso Mínimo Vital (IMV).

La gestión de esta prestación, se llevó a cabo mediante un equipo de voluntarios que se incorporaron a la asociación durante el confinamiento. La pandemia puso de manifiesto la poliédrica realidad de la brecha digital padecida por un buen número de vecinas y vecinos de nuestro barrio. A partir de esta experiencia se ha puesto en marcha, como hemos dicho anteriormente, un proyecto de acceso a la administración digital.

\section{CONCLUSIONES}

El trabajo en red entre movimiento vecinal, tercer sector y administraciones públicas, ha sido la clave para reducir el sufrimiento entre el vecindario de Zofío. Lejos de prescindir de los Servicios Sociales y sus profesionales, ya sean en la atención primaria o en los centros educativos, consideramos que son piezas esenciales, al aportar criterios técnicos. De la misma manera, el tercer sector y el movimiento vecinal aportan una red de proximidad al vecindario y una respuesta rápida y poco burocratizada. Creemos firmemente que estas fórmulas de trabajo pueden dar pasa a formas más estables y complejas de trabajo comunitario que, lejos de incrementar costes, son la única respuesta eficaz para muchos problemas sociales.

\section{REFERENCIAS}

Alianza Social en Defensa del Sistema Público de Servicios Sociales (2020). Servicios Sociales y COVID-19 en la Comunidad de Madrid. Segunda ola de la pandemia. Recuperado de: https://bit.ly/2STIse9

Artigas, M. y Días, S. (3 de junio de 2020). Apoyo Mutuo Usera: la sociedad civil responde a la pandemia. Cuarto Poder. Recuperado de: https://bit.ly/2ReVanq

Asociación Estatal de Directores y Gerentes en Servicios Sociales. (2019). Desarrollo de los Servicios Sociales por Comunidades Autónomas. Índice DEC 2018. Recuperado de: https://bit.ly/3wfxKx3

Ayuntamiento de Madrid (2018). Metodología para la elaboración del índice de vulnerabilidad territorial de barrios y distritos de Madrid y ranking de vulnerabilidad. Recuperado de: https://bit.ly/2Q1G3rB 
Ayuntamiento de Madrid. (2020). Madrid 2020: Diagnóstico social de la crisis por COVID-19. Recuperado de: https://bit.ly/3on0ZuN

Benach, J. y Muntaner, C. (2005). Aprender a mirar la salud. Cómo la desigualdad social daña nuestra salud. Barcelona. El Viejo Topo.

Bonet, J. (2012). El territorio como espacio de radicalización democrática: una aproximación crítica a los procesos de participación ciudadana en las políticas urbanas de Madrid y Barcelona. Athenea Digital. Vol. (12), Nº 1, 15-28. Recuperado de: https://bit.ly/2Uv85mj

Bustos, B. (2006). El protagonismo femenino en las asociaciones vecinales de Alicante durante los años sesenta ( ${ }^{\circ}$ 5), 289-296. Recuperado de: https://bit.ly/2ReEcW3

Calzada, I. (Coord.) (2020). Instituto Nacional de Administraciones Públicas. Los servicios sociales ante la pandemia: retos, desafíos y respuestas hacia la nueva normalidad. Recuperado de: https://bit.ly/3bpXuib

Cañedo, M. (2011). Discursos vecinales sobre la inseguridad ciudadana y políticas de rehabilitación urbanística: el caso de los «antiguos vecinos» y la ARI-Lavapiés (Madrid) desde una perspectiva antropológica. Scripta Nova, (No. 15), 348-386. Recuperado de: https://bit.ly/2QmPAi9

Caprarella, M. (2016). Crónica de una capital en tránsito. Crisis económica, luchas ciudadanas y cambio cultural en Madrid (1975-1985). Postmetrópolis.

Cáritas Española (2021). Un año acumulando crisis. La realidad de las familias acompañadas por Cáritas en enero de 2021. Observatorio de la Realidad Social. La crisis del COVID-19, ( $N^{\circ} 3$ ). Recuperado de: https://bit.ly/3ojXAgt

Castells, M. (1986). La ciudad y las masas. Alianza Editorial.

Díaz, L. B. (1999). Asociaciones vecinales. Fortalecimiento de la sociedad civil. Consolidación del estado democrático (los casos de España y Colombia). [Tesis doctoral, Universidad Complutense de Madrid]. Recuperado de: https://bit.ly/3yhhQUE

Escudero, F. (2020). La lucha por el barrio y el medio ambiente. Movimientos vecinales y ecologistas. Otra cara de la lucha contra la Dictadura (1970-1980). Historia Actual Online, ( $\left.N^{o} .52\right), 113-128$. Recuperado de: https://bit.ly/3fpTgrS

Federación Regional de Asociaciones de Vecinos de Madrid (2020). Dinamiza tu cuarentena: redes vecinales de apoyo ante el Covid-19. Recuperado de: https://bit. ly/2RSQLq2

FOESSA (2019). Informe sobre exclusión social y desarrollo en Madrid. Recuperado de: https://bit.ly/2ReEcW3

Franco, L. (16 de junio de 2020). Las asociaciones de vecinos de Madrid duplican sus entregas de comida en un mes. [Versión digital] El País. Recuperado de: https:// bit.ly/3oleV8F

Gallardo, J. J. (2015). Re-ocupación y/o re-construcción de estructuras urbanas en desuso y su conexión con el espacio público y el paisaje. [Tesis doctoral, Universidad Politécnica de Madrid]. Recuperado de: https://bit.ly/3eRZzp8

García, N. (2021). Percepción ciudadana de los problemas medioambientales y de sostenibilidad urbana en contextos metropolitanos. Análisis desde las Agendas 21 de cuatro municipios de la Región de Madrid (ESPAÑA). [Tesis doctoral, Universidad Nacional de Educación a Distancia]. Recuperado de: https://bit.ly/3uSm3Mo 
Gómez, A. B. (2017). Problemática vecinal y batalla ciudadana en la transición democrática en Jaén: 1975-1985. Antropología Experimental ( $\left.N^{\circ} 17\right), 195-214$. Recuperado de: https://bit.ly/3eVxldn

González, E. V., Arredondo R. y Rodríguez, C. (2019). Consejo General del Trabajo Social. Tercer informe sobre los Servicios Sociales en España. Recuperado de: https://bit.ly/3hszgrp

Hedo, I. (2010). Acción colectiva vecinal en el tardofranquismo: El caso de Rekalde. Historia y política. ( $\mathrm{N}^{\circ} 23$ ), págs. 275-296. Recuperado de: https://bit.ly/3tOXbnd

Jiménez, C. (2013). Espacios de informalidad y movimientos sociales en Madrid, 1968-2011. Bitácora Urbano-Territorial, (Vol. 23, No. 2), págs. 23-47. Recuperado de: https://bit.ly/3tSO30S

Limón, P. (2015). Un barrio para gobernarlos a todos: Gentrificación, producción de globalidad y barrionalismo en Hortaleza (Madrid) y Poblenou (Barcelona) (19922014). [Tesis doctoral, Universidad Complutense de Madrid]. Recuperado de: https://bit.ly/3eShwUo

Manahan, M., Villanueva, E., Alegado J. E. y Morón-Cara, Y. (2020). (Re)construir servicios públicos frente a la gobernanza neoliberal: prácticas de sistemas asociativos en torno al agua. Relaciones Internacionales, ( $\left.\mathrm{N}^{\mathbf{0}} 45\right)$, 205-226. Recuperado de: https://bit.ly/3fnAX6G

Montañés, M. y Gómez, M. C. (2020). El asociacionismo y la participación vecinal luchando por la vivienda, haciendo ciudad. Hábitat y Sociedad, (N. $\left.{ }^{\circ} 13\right), 287-298$. Recuperado de: https://bit.ly/3eNdYTE

Morell, C. G. (2012). Mujeres y vecinas en la transición: el caso de Valladolid, 1970-1986. Cuadernos Kóre., Nº. 6, 2012, 148-175. Recuperado de: https://bit.ly/33NS9wM

Morell, C. G. (2013). Un movimiento social urbano contra los especuladores: La Rondilla frente a la Imperial S.L. Revista Historia Autónoma, $N^{o}$. 3, 129-142. Recuperado de: https://bit.ly/3ydUgI9

Navarro, V. (2006). El subdesarrollo social de España. Causas y consecuencias. Anagrama.

Negro, M. (25 de febrero de 2021). Los servicios sociales de 9 distritos están por debajo del mínimo que fija el Ayuntamiento de Madrid. Edición digital de El País. Recuperado de: https://bit.ly/3bwThsX

Rochet, A. (18 de mayo de 2020). «Despensas solidarias» para acabar con el hambre en Madrid. Madridiario. Recuperado de: https://bit.ly/2Re9i0b

Ruano, J. M., Iglesias, E. y Polo, C. (2019). El Madrid vivido: los problemas urbanos desde la perspectiva de la ciudadanía en el contexto del turismo de masas. Boletín de la Asociación de Geógrafos Españoles, $N^{\circ}$. 83, págs. 110-132. Recuperado de: https://bit.ly/33Mwrcz.

Torres, V. (14 de abril de 2020). Los trabajadores sociales, ante la doble crisis de los que ya estaban en crisis. [Versión digital] El País. Recuperado de: https://bit. ly/3okxl9C

Uceda, P. (2016). La ciudad desequilibrada. El derecho a la ciudad en los barrios vulnerables de Madrid. [Tesis doctoral, Universidad Complutense de Madrid]. Recuperado de: https://bit.ly/3waM1e6 